\title{
Differential composition of gut microbiota among healthy volunteers, morbidly obese patients and post-bariatric surgery patients
}

\author{
FU-GANG WANG ${ }^{1}$, RI-XING BAI ${ }^{1}$, WEN-MAO YAN ${ }^{1}$, MING YAN $^{1}$, LING-YUE DONG ${ }^{2}$ and MAO-MIN SONG \\ ${ }^{1}$ Department of General Surgery, Beijing Tiantan Hospital, Capital Medical University, Beijing 100050; \\ ${ }^{2}$ Department of Cell Biology, Basic Medical College, Capital Medical University, Beijing 100069, P.R. China
}

Received June 23, 2018; Accepted December 31, 2018

DOI: $10.3892 /$ etm.2019.7200

\begin{abstract}
The modulation of the gut microbiota was recently deemed one of the mechanisms responsible for the excellent outcomes of bariatric surgery. However, to date, only few studies have assessed this, and they have high heterogeneity. In the present study, next-generation 16S ribosomal DNA amplicon sequencing was used to characterize the gut microbiota of healthy volunteers, as well as patients prior to and after sleeve gastrectomy (SG) or Roux-en-Y gastric bypass (RYGB). Significant differences in $\alpha$ diversity, $\beta$ diversity and species were identified between the different groups/time-points. The results demonstrated excellent outcomes of SG and RYGB. The $\beta$ diversity was lower in healthy volunteers compared with that in morbidly obese patients with or without type 2 diabetes mellitus. At 3 months after SG, the $\alpha$ diversity was increased and the $\beta$ diversity was decreased. The abundance of certain species changed significantly after SG and RYGB. It was also revealed that the abundance of certain microbes was significantly correlated with the body mass index, fasting blood glucose and glycosylated haemoglobin. It may be concluded that bariatric surgery may cause obvious alterations in the gut microbiota and compared with healthy volunteers and obese patients
\end{abstract}

Correspondence to: Dr Mao-Min Song, Department of General Surgery, Beijing Tiantan Hospital, Capital Medical University, 6 Tiantanxili Road, Beijing 100050, P.R. China

E-mail: songmaomin@163.com

Professor Ling-Yue Dong, Department of Cell Biology, Basic Medical College, Capital Medical University, 10 Xitoutiao Road, Beijing 100069, P.R. China

E-mail: lingyued@ccmu.edu.cn

Abbreviations: SG, sleeve gastrectomy; RYGB, Roux-en-Y gastric bypass; T2DM, type 2 diabetes mellitus; Glu, fast blood glucose; $\mathrm{GHb}$, glycosylated haemoglobin

Key words: morbid obesity, type 2 diabetes mellitus, bariatric surgery, gut microbiota, sleeve gastrectomy, Roux-en-Y gastric bypass without bariatric surgery, the microbiota composition of post-bariatric surgery has unique characteristics. However, studies with a larger cohort and longer follow-up may be required to confirm these results.

\section{Introduction}

At present, bariatric surgery is the only method available to treat obesity and associated comorbidities with lasting efficacy (1). Compared with non-surgical interventions, bariatric surgery achieves a greater improvement with regard to weight loss outcomes and obesity-associated comorbidities, including type 2 diabetes mellitus (T2DM), hypertension and hyperlipemia (2). Sleeve gastrectomy (SG) and Roux-en-Y gastric bypass (RYGB) are the two most common procedures used to treat obesity worldwide (3). SG is a restrictive and technically less complex procedure than RYGB, which is a procedure that involves a restrictive and malabsorptive mechanism (4). The potential mechanisms underlying the excellent outcomes of bariatric surgery include the following: Restriction of dietary intake and nutritional supply (5), modulation of gut hormones (6) and alterations of inflammatory factors (7).

Disorders of gut microbiota are associated with numerous diseases (8-10). Indeed, studies have demonstrated different patterns of gut microbiota among healthy volunteers, obese patients and T2DM patients $(11,12)$. In recent years, modulation of gut microbiota has been deemed another important mechanism underlying the exceptional outcomes of bariatric surgery (13). Tremaroli et al (14) separately colonized germ-free mice with stool from patients after bariatric surgery and obese controls. They observed a $26-43 \%$ elevation of fat deposition of mice colonized with stools from obese controls 9.4 years following bariatric surgery, and they concluded that the changes in the gut microbiota may be one of the factors responsible for the excellent outcomes of bariatric surgery (14). Few studies have explored the alterations of the gut microbiota after bariatric surgery, and the populations used in these studies were too small, and the results contained high heterogeneity (14-16). For this reason, the present study performed a similar analysis of the effect of bariatric surgery on the gut microbiota with the aim of complementing the previously reported data. 


\section{Patients and methods}

Patient inclusion and exclusion. A total of 20 healthy volunteers and 26 obese patients with or without T2DM [19 patients receiving SG and 7 patients receiving RYGB] were recruited for the present study from August 2017 to May 2018 at Beijing Tiantan Hospital. The healthy volunteers were age and sex matched with the obese patients intending to receive SG. At three months after bariatric surgery, a follow-up examination was performed and the stool was collected from 8 patients receiving $\mathrm{SG}$ and 3 patients receiving RYGB. All patients and healthy volunteers provided written informed consent and the present study was approved by the ethics committee of Bejing Tiantan Hospital (Beijing, China). The patient selection criteria for RYGB were as follows: i) Patients were adults, with an age ranging from 18 to 65 years; ii) patients had relatively severe T2DM (duration of T2DM, $\geq 2$ years and T2DM requiring insulin treatment); and iii) the patient's desire to receive RYGB. The inclusion criteria for SG were as follows: i) Patients were adults, with the age ranging from 18 to 65 years; ii) patients had relatively minor T2DM (duration of T2DM, <2 years or T2DM requiring anti-diabetic or dietary therapy); and iii) patients had a body mass index (BMI) of $>28 \mathrm{~kg} / \mathrm{m}^{2}$. For the healthy volunteers, the inclusion criteria were as follows: i) Volunteers were adults, with the age ranging from 18 to 65 years; ii) a BMI ranging from 18 to $24 \mathrm{~kg} / \mathrm{m}^{2}$; iii) no T2DM. The following exclusion criteria were applied to all patients and healthy volunteers: i) Antibiotic use within the previous 3 months; ii) pregnancy or the intention of conceiving; iii) previous gastrointestinal surgery; iv) serious mental disease; v) diarrhea within the previous month; vi) use of proton pump inhibitors within the previous month; vii) alcohol use of $>25 \mathrm{~g} /$ day; viii) special dietary habits.

\section{Surgical procedures}

$R Y G B$. First, the gastric pouch was created with a volume of $\sim 20 \mathrm{ml}$ and the jejunum was then transected $100 \mathrm{~cm}$ below the ligament of Treitz. Subsequently, $100 \mathrm{~cm}$ of the Roux limb was anastomosed to the gastric pouch (antecolic gastrojejunostomy), and $100 \mathrm{~cm}$ of the biliopancreatic limb was subsequently anastomosed in an end-to-side fashion to the distal Roux limb.

SG. A 36Fr orogastric bougie was passed through the anterior wall of the stomach and then cut. The lesser curvature was closed 5-6 cm away from the pylorus towards the cardia with a linear cut stapler.

Stool sample collection and grouping. In total, 57 stool samples were collected from the 20 healthy volunteers and 26 patients at 3 days pre-operatively, and from 11 patients at 3 months post-operatively. The 57 samples were divided into 5 groups as follows: Control group ( $\mathrm{CO}, \mathrm{n}=20)$, patients prior to $\mathrm{SG}$ (SG0, n=19), patients prior to RYGB (RYGB0, n=7), patients after SG (SG3, n=8) and patients after RYGB (RYGB3, n=3). These patients who were lost to follow up following bariatric surgery could not be paired prior to and following surgery, while those patients who were followed up successfully could be. This meant that patients were not strictly paired between
SG0 and SG3 or between RYGB0 and RYGB3. The stool samples were self-collected in sterile stool containers and within $2 \mathrm{~h}$, they were stored at $-80^{\circ} \mathrm{C}$ until DNA extraction.

\section{Sequencing}

Extraction of genomic DNA. Total genomic DNA was extracted from the samples using the cetyl trimethylammonium bromide method (17). DNA concentration and purity were assessed on $1 \%$ agarose gels. According to the concentration in the extract, the DNA was diluted to $1 \mathrm{ng} / \mu \mathrm{l}$ using sterile water.

Amplicon generation. Distinct regions of $16 \mathrm{~S}$ ribosomal RNA gene (16S V4) were amplified using a specific primer pair (515 forward, 5'-GTGCCAGCMGCCGCGGTAA-3'; 806 reverse, 5'-GGACTACHVGGGTWTCTAAT-3'; M, H and V represent degenerate bases) with the barcode. All PCRs were performed with Phusion ${ }^{\circledR}$ High-Fidelity PCR Master Mix (New England BioLabs, Inc., Ipswich, MA, USA) according to the manufacturer's protocol. The Bio-rad T100 PCR apparatus was utilized (Bio-Rad Laboratories, Inc., Hercules, CA, USA).

PCR product mixing and purification. The PCR products were mixed with the same volume of $1 \mathrm{X}$ loading buffer (Thermo Fisher Scientific, Inc., Waltham, MA, USA; containing SYBR green) and subjected to electrophoresis in a $2 \%$ agarose gel for detection. PCR products from various samples were mixed at equal amounts to ensure the evenness of products between samples. Subsequently, the mixtures of PCR products were purified with a GeneJETTM Gel Extraction kit (Thermo Fisher Scientific, Inc.).

Library preparation and sequencing. Sequencing libraries were generated using an Ion Plus Fragment Library kit 48 rxns (Thermo Fisher Scientific, Inc.) according to the manufacturer's protocols. The library quality was assessed using a Qubit ${ }^{\circledR} 2.0$ Fluorometer (Thermo Fisher Scientific, Inc.). Finally, the library was sequenced on the Ion S5 ${ }^{\mathrm{TM}}$ XL platform (Thermo Fisher Scientific, Inc.) and 400/600-bp single-end reads were generated.

\section{Data analysis}

Quality control of single-end reads. Single-end reads were assigned to samples based on their unique barcode and were truncated by removing the barcode and primer sequences. Quality filtering on raw reads was performed under specific filtering conditions to obtain high-quality clean reads according to the Cutadapt quality control process (18). The reads were compared with a reference database (19) using the UCHIME algorithm (20) to detect chimeric sequences, which were then removed (21). Ultimately, clean reads were obtained.

Operational taxonomic units (OTU) cluster and species annotation. Sequence analyses were performed using Uparse software (Version 7.0.1001) (22). Sequences with $\geq 97 \%$ similarity were considered to be identical OTUs and deemed to be of one certain species. Representative sequences for each OTU were screened for further annotations. For each representative sequence, the Silva Database (19) was used based on the Mothur algorithm to annotate taxonomic information. 
Table I. Clinical characteristics of the study population.

\begin{tabular}{lcccccc}
\hline Parameter & CO $(\mathrm{n}=20)$ & SG0 $(\mathrm{n}=19)$ & RYGB0 $(\mathrm{n}=7)$ & SG3 $(\mathrm{n}=8)$ & RYGB3 (n=3) & P<0.05 comparisons \\
\hline Age (years) & $31.5 \pm 10.3$ & $32.9 \pm 7.1$ & $45.7 \pm 9.4$ & $33.3 \pm 6.5$ & $40.3 \pm 6.8$ & CO vs. RYGB0; SG0 vs. RYGB0; \\
Females & $14(70 \%)$ & $16(84 \%)$ & $4(57 \%)$ & $8(100 \%)$ & $3(100 \%)$ & \\
T2DM & & $14(74 \%)$ & $7(100 \%)$ & $0(0 \%)$ & $2(67 \%)$ & SG0 vs. SG3 \\
BMI $\left(\mathrm{kg} / \mathrm{m}^{2}\right)$ & $22.4 \pm 1.4$ & $41 \pm 5.6$ & $30 \pm 4.7$ & $30.6 \pm 3.8$ & $26.2 \pm 2$ & CO vs. SG0; CO vs. RYGB0; \\
& & & & & & SG0 vs. SG3 \\
GHb (\%) & & $7.4 \pm 2$ & $7.8 \pm 1.6$ & $5.6 \pm 0.4$ & $7.4 \pm 1.4$ & SG0 vs. SG3; \\
Glu (mmol/l) & & $5.7 \pm 2.2$ & $7.4 \pm 3.4$ & $4.9 \pm 0.9$ & $9.3 \pm 2.3$ & \\
\hline
\end{tabular}

As the healthy volunteers refused invasive testing, T2DM, GHb and Glu for the CO group were not provided. In the SG0 group, 14 out of 19 patients had T2DM. At 3 months after SG, only 8 patients were available for follow-up, 4 of which had pre-operative T2DM, and all of them achieved complete T2DM remission (complete remission rate, 100\%). In the RYGB0 group, 7 patients had T2DM. At 3 months after RYGB, only 3 patients were available for follow-up and of these, 1 achieved complete T2DM remission, while the other 2 patients achieved partial T2DM remission (complete remission rate, 33\%). T2DM, type 2 diabetes mellitus; BMI, body mass index; GHb, glycosylated haemoglobin; Glu, blood glucose; BMI, body mass index; CO, control group; SG, sleeve gastrectomy; RYGB, Roux-en-Y gastric bypass; SG0/3, group prior to/3 months after SG.

To explore the phylogenetic association of different OTUs, as well as the differences in the dominant species in different samples (groups), multiple sequence alignments were performed using MUSCLE software version 3.8.31 (23). The abundance information of the OTUs was normalized according to a standard sequence number corresponding to the sample with the fewest sequences. Subsequent analyses of $\alpha$ diversity and $\beta$ diversity were all performed based on this output normalized data.

$\alpha$ diversity. $\alpha$ diversity is applied when analyzing the complexity of species diversity for a sample through 6 indices, including Observed-species (http://scikit-bio.org/docs/ latest/generated/generated/skbio.diversity.alpha.observed_otus. html?highlight=observed\#skbio.diversity.alpha.observed_otus), Chao1 (http://www.mothur.org/wiki/Chao), Shannon (http://www.mothur.org/wiki/Shannon), abundance-based coverage estimator (ACE) (http://www.mothur.org/wiki/Ace), Good-coverage(https://en.wikipedia.org/wiki/Coverage_(genetics)) and phylogenetic diversity (PD)_whole_tree (http://scikit-bio. org/docs/latest/generated/generated/skbio.diversity.alpha.faith_ pd.html?highlight=pd\#skbio.diversity.alpha.faith_pd). All indices in the present samples were calculated with QIIME (version 1.7.0; http://qiime.org/) and displayed with R software (version 2.15.3). The rarefaction curves were generated by using the ggplot2 package for R.

Characteristics of each of the indices used. Observed-species is a common index reflecting the variety of species in one sample. Chaol includes the low-abundance species in the formula, so this index is able to better reflect the existence of low-abundance species. Shannon's diversity index not only reflects the variety of species but also takes into account the abundance of each species. The ACE index not only reflects the variety of species but also considers the probability that one species exists in one certain sample. The PD_whole_tree index reflects the genetic similarity between species, and further genetic associations between species in a sample indicate a higher PD_whole_tree index. In other words, the evolutionary distance between species in a sample is further and the PD_whole_tree index is higher.

$\beta$ diversity. $\beta$ diversity, on weighted and unweighted unifrac, was calculated with QIIME software (version 1.7.0). The $\beta$ diversity reflects the compositional difference between samples in one certain group. Weighted unifrac includes abundance and variety of species in the process of calculation, while unweighted unifrac includes only variety. Non-metric multidimensional scaling (NMDS) analysis was performed using the vegan package for $\mathrm{R}$. The linear discriminant analysis effect size (LEfSe) with default parameters was used to identify species with significant differences between groups (24). The statistical method applied using LEfSe software (version 1.0 http://huttenhower.sph.harvard.edu/galaxy/) was the Kruskal-Wallis test. In order to identify those microbes with beneficial or harmful effects on surgical outcomes, a spearman correlation analysis was performed between species and clinical indexes by using the psych package for $\mathrm{R}$. Spearman correlation analysis was used in all experimental groups (SG0, SG3, RYGB0 and RYGB3). The control group was not included in spearman correlation analysis due to the absence of clinical data, including GHb and Glu. $\mathrm{P}<0.05$ was considered to indicate statistical significance.

Data analysis of clinical characteristics. The data analysis of clinical characteristics was performed using SPSS 24.0 software (IBM Corp., Armonk, NY, USA). Continuous data was compared using an unpaired t-test (2 groups) or one-way ANOVA (multiple groups) followed by an LSD post-hoc test. Categorical data was compared using a Fischer's exact test.

\section{Results}

Clinical characteristics of the study population. The clinical characteristics of the 5 groups are presented in Table I. The results indicated favorable BMI reduction and T2DM 


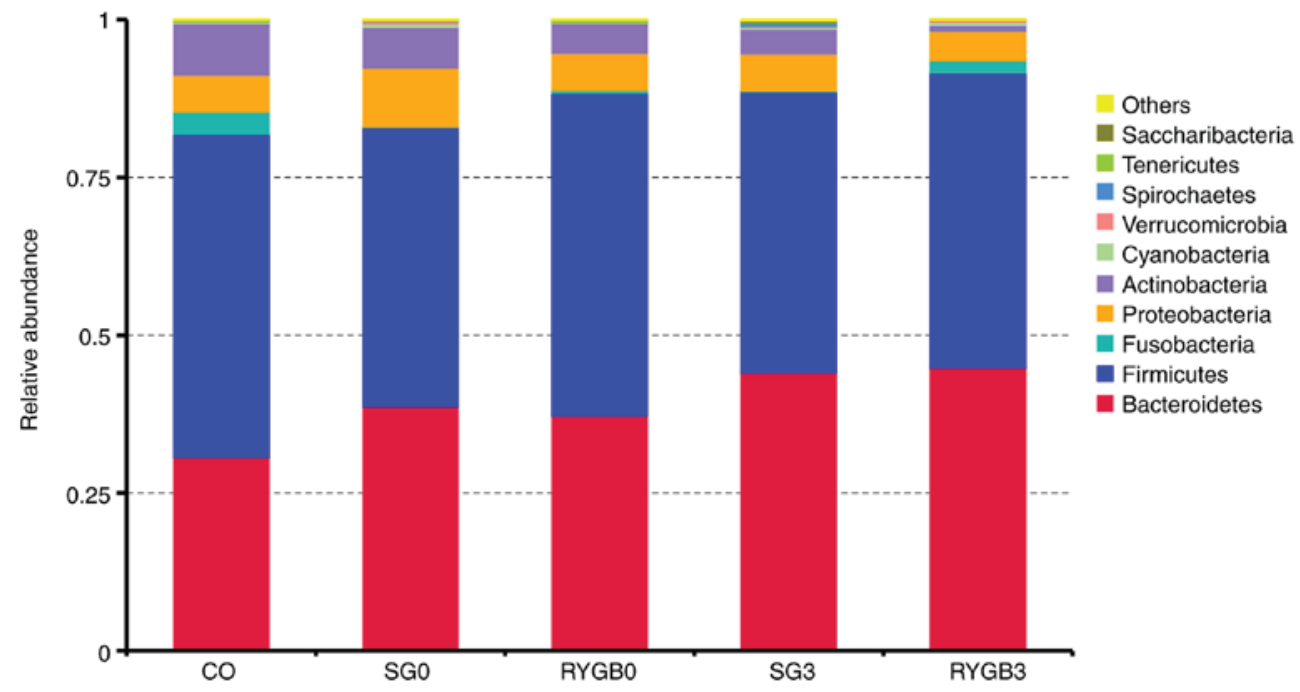

Figure 1. Top 10 species with the highest relative abundance of the 5 groups at the level of phylum. The top 5 phyla were Bacteroidetes, Firmicutes, Fusobacteria, Proteobacteria and Actinobacteria. No statistically significant differences were identified among species at the level of phylum. However, a decreasing trend of Bacteroidetes and an increasing trend of Fusobacteria was determined in the CO group compared with that in the SG0 group (P=0.28 for Bacteroidetes and $\mathrm{P}=0.32$ for Fusobacteria) and RYGB0 group ( $\mathrm{P}=0.48$ for Bacteroidetes and $\mathrm{P}=0.37$ for Fusobacteria). An increasing trend of Bacteroidetes was identified in the $\mathrm{SG} 3$ group $(\mathrm{P}=0.49)$ and RYGB3 group $(\mathrm{P}=0.53)$ compared with that in the $\mathrm{SG} 0$ group and the RYGB0 group. CO, control group; $\mathrm{SG}$, sleeve gastrectomy; RYGB, Roux-en-Y gastric bypass; SG0/3, group prior to/3 months after SG.

remission of SG and RYGB. The results revealed a markedly lower BMI, T2DM rate and GHb in the SG3 group compared with the SG0 group $(\mathrm{P}<0.05)$. However, due to the small sample size, no statistically significant differences were observed in BMI, T2DM rate, GHb and Glu between the RYGB0 group and the RYGB3 group. In the SG0 group, 14 of the 19 patients included had T2DM. At 3 months following SG, only 8 patients were available for follow-up, 4 of which exhibited pre-operative T2DM, with all of them achieving complete T2DM remission (complete remission rate, 100\%). In the RYGB0 group, 7 patients had T2DM. At 3 months following RYGB, only 3 patients were available for follow-up, and of these, 1 achieved complete T2DM remission, while the other 2 patients achieved partial T2DM remission (complete remission rate, 33\%). In general, obese patients with a lower BMI, an older age and a more severe T2DM tend to be selected to perform RYGB. As for SG, obese patients with a higher BMI, younger age and relatively less severe T2DM tended to be selected. The relatively lower BMI, older age and more severe T2DM in the RYGB0 group compared with the SG0 group is indicated in Table I. The indexes for determining the severity of T2DM were not the results of glucose and insulin tests, but the duration of T2DM and mode of treatment; in addition, the insulin test is not a routine examination during post-operative follow-up at our institution. For this reason, T2DM was not graded according to glucose and insulin test results. It may be observed from Table I that the female gender ratios in the CO, SG0, RYGB0, SG3 and RYGB3 groups were 70, 74, 57, 100 and $100 \%$, respectively.

Relative abundance. On the basis of the results of the species annotation, the distribution among the 5 groups at the phylogenetic level was determined. As displayed in the bar graph in Fig. 1, the top 5 phyla were Bacteroidetes, Firmicutes, Fusobacteria, Proteobacteria and Actinobacteria. The detailed proportions of the top 10 phyla in the 5 groups are listed in Table II. In the horizontal comparison, a slightly lower proportion of Bacteroidetes and a slightly higher proportion of Fusobacteria were identified in the CO group compared with those in the $\mathrm{SG} 0$ group $(\mathrm{P}=0.28$ for Bacteroidetes and $\mathrm{P}=0.32$ for Fusobacteria) and the RYGB0 group ( $\mathrm{P}=0.48$ for Bacteroidetes and $\mathrm{P}=0.37$ for Fusobacteria). In the longitudinal comparison, a slightly higher proportion of Bacteroidetes was identified in the SG3 group $(\mathrm{P}=0.49)$ and the RYGB3 group $(\mathrm{P}=0.53)$ compared with that in the $\mathrm{SG} 0$ group and the RYGB0 group, respectively.

$\alpha$ diversity. The $\alpha$ diversity of the 5 groups was calculated in 6 different algorithms, including ACE (Fig. 2A), Chaol (Fig. 2B), Goods_coverage, Observed_species (Fig. 2C), Shannon (Fig. 2D) and PD_whole_tree (Fig. 2E). No statistically significant differences regarding the $\alpha$ diversity were identified among the CO, SG0 and RYGB0 groups. Of note, a significantly higher $\alpha$ diversity was revealed in the SG3 group compared with that in the SG0 group (Fig. 2). Rarefaction curves were generated according to Chaol (Fig. 3A), Observed_species (Fig. 3B), PD_whole_tree (Fig. 3C) and Shannon (Fig. 3D). The rarefaction curves were obtained from multiple samplings of the same individuals and with a variation in the depth of the sequencing; this was used to visually demonstrate the reasonability of the amount of sequencing data. The $\mathrm{x}$-axis of the rarefaction curves represented the amount of sequencing data extracted from one sample and the $y$-axis represented the number of species. In Fig. 3 , the 5 lines of the 5 groups gradually became flat, which indicated that the amount of sequencing data was appropriate. In other words, if the amount of sequencing data had been further increased, no new species would have been found. The rarefaction curves also reflected the $\alpha$ diversity, and the results were consistent with those in Fig. 2.

$\beta$ diversity. The $\beta$ diversity of the 5 groups was calculated using the ANOSIM algorithm. Analysis using unweighted_unifrac 
Table II. Detailed proportion of the top 10 phyla in the 5 groups.

\begin{tabular}{|c|c|c|c|c|c|}
\hline Taxonomy & $\mathrm{CO}$ & SG0 & RYGB0 & SG3 & RYGB3 \\
\hline Bacteroidetes & 0.306591 & 0.387295 & 0.372221 & 0.440632 & 0.448629 \\
\hline Firmicutes & 0.512184 & 0.441977 & 0.511654 & 0.444814 & 0.467192 \\
\hline Fusobacteria & 0.035732 & 0.000883 & 0.004699 & 0.001771 & 0.019554 \\
\hline Proteobacteria & 0.058096 & 0.093562 & 0.058359 & 0.058849 & 0.046483 \\
\hline Actinobacteria & 0.082309 & 0.064907 & 0.047962 & 0.039593 & 0.010045 \\
\hline Cyanobacteria & 0.000983 & 0.005560 & 0.000256 & 0.001785 & 0.004170 \\
\hline Verrucomicrobia & 0.000976 & 0.003613 & 0.000915 & 0.002588 & 0.003688 \\
\hline Spirochaetes & 0.000051 & 0.000820 & 0.000107 & 0.005384 & 0.000018 \\
\hline Tenericutes & 0.002878 & 0.000035 & 0.003570 & 0.000318 & 0.000089 \\
\hline Saccharibacteria & 0.000096 & 0.000355 & 0.000084 & 0.002578 & 0.000054 \\
\hline Others & 0.000103 & 0.000992 & 0.000172 & 0.001688 & 0.000080 \\
\hline
\end{tabular}

None of the species of phyla exhibited a statistically significant difference in its proportion among the different groups. However, a slightly lower proportion of Bacteroidetes and a slightly higher proportion of Fusobacteria were identified in the CO group compared with those in the SG0 group $(\mathrm{P}=0.28$ for Bacteroidetes and $\mathrm{P}=0.32$ for Fusobacteria) and RYGB0 group ( $\mathrm{P}=0.48$ for Bacteroidetes and $\mathrm{P}=0.37$ for Fusobacteria). A slightly higher proportion of Bacteroidetes was identified in the SG3 group ( $\mathrm{P}=0.49)$ and RYGB3 group ( $\mathrm{P}=0.53)$ compared with the SG0 group and the RYGB0 group, respectively. CO, control group; SG, sleeve gastrectomy; RYGB, Roux-en-Y gastric bypass; SG0/3, group prior to/3 months after SG.
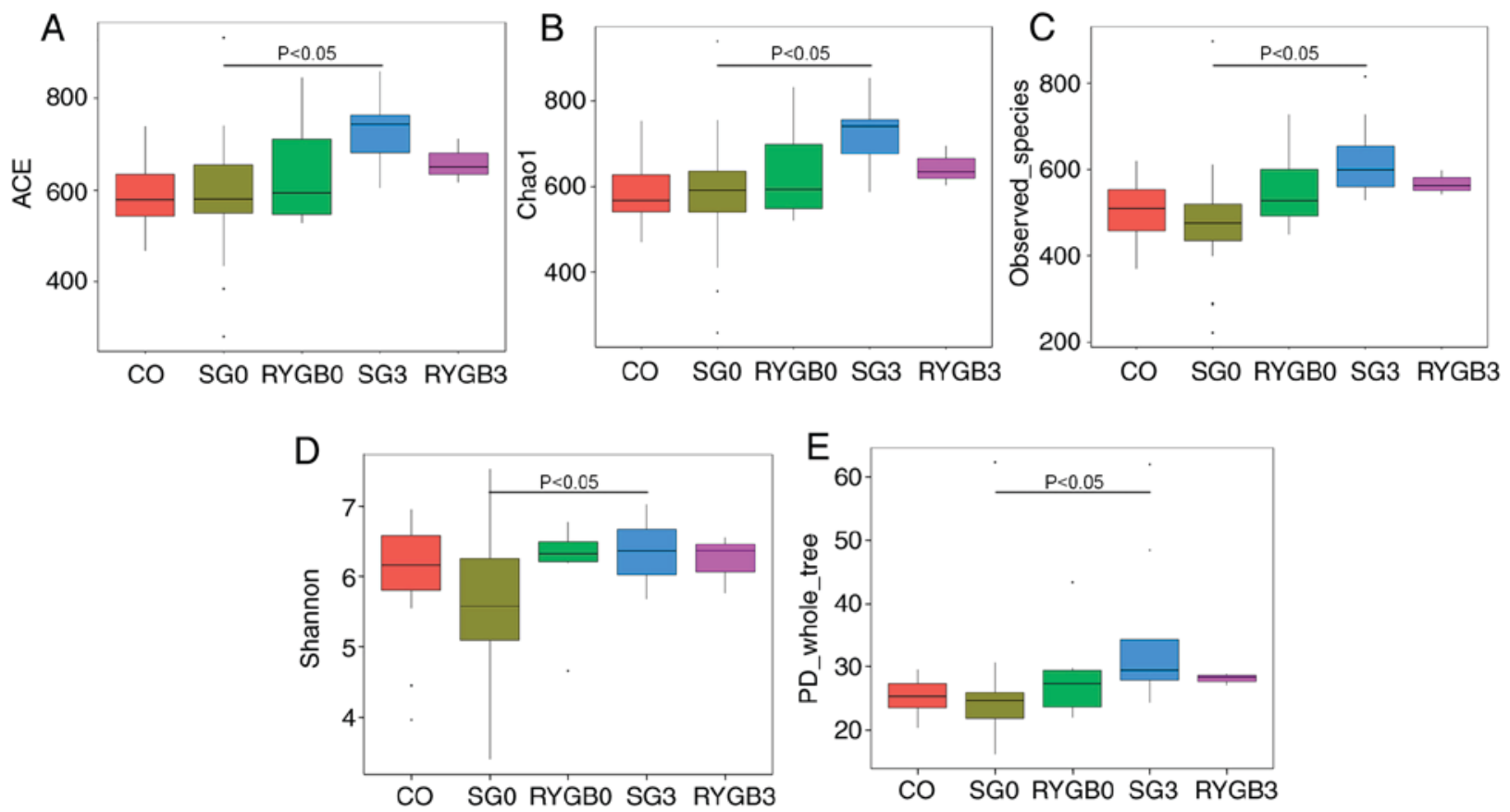

Figure 2. $\alpha$ diversity in 5 groups determined by 6 different algorithms. (A) ACE; (B) chao1; (C) observed_species; (D) Shannon; (E) PD_whole_tree. No significant difference was identified in $\alpha$ diversity among the CO group, SG0 group and RYGB0 group. An obviously higher $\alpha$ diversity was obtained in the SG3 group compared with that in the SG0 group $(\mathrm{P}<0.05)$. A tendency of higher $\alpha$ diversity may be distinguished in the RYGB3 group compared with that in the RYGB0 group from A-C. The horizontal lines inside the boxes indicate the median, whereas the lower lines and upper lines of the boxes indicate the 25th and the 75th percentiles, respectively. The upper and lower ends of the vertical lines represent the maximum and minimum points of data, respectively. The dots outside the boxes indicate the outliers (an observation point whose distance from mean value is greater than double standard deviations). CO, control group; SG, sleeve gastrectomy; RYGB, Roux-en-Y gastric bypass; SG0/3, group prior to/3 months after SG; ACE, abundance-based coverage estimator; $\mathrm{PD}$, phylogenetic diversity.

revealed an obviously higher $\beta$ diversity in the SG0 group compared with that in the $\mathrm{CO}$ group $(\mathrm{P}<0.05$; Fig. 4A). Furthermore, analysis with weighted_unifrac indicated a significantly higher $\beta$ diversity in the SG0 group and the RYGB0 group compared with that in the CO group $(\mathrm{P}<0.05)$, as well as an obviously lower $\beta$ diversity in the SG3 group compared with that in the SG0 group $(\mathrm{P}<0.05$; Fig. 4B). The heterogeneity in the community composition of the 5 groups was presented in a two-dimensional way using an NMDS reduced-dimension graph. The community composition of the 

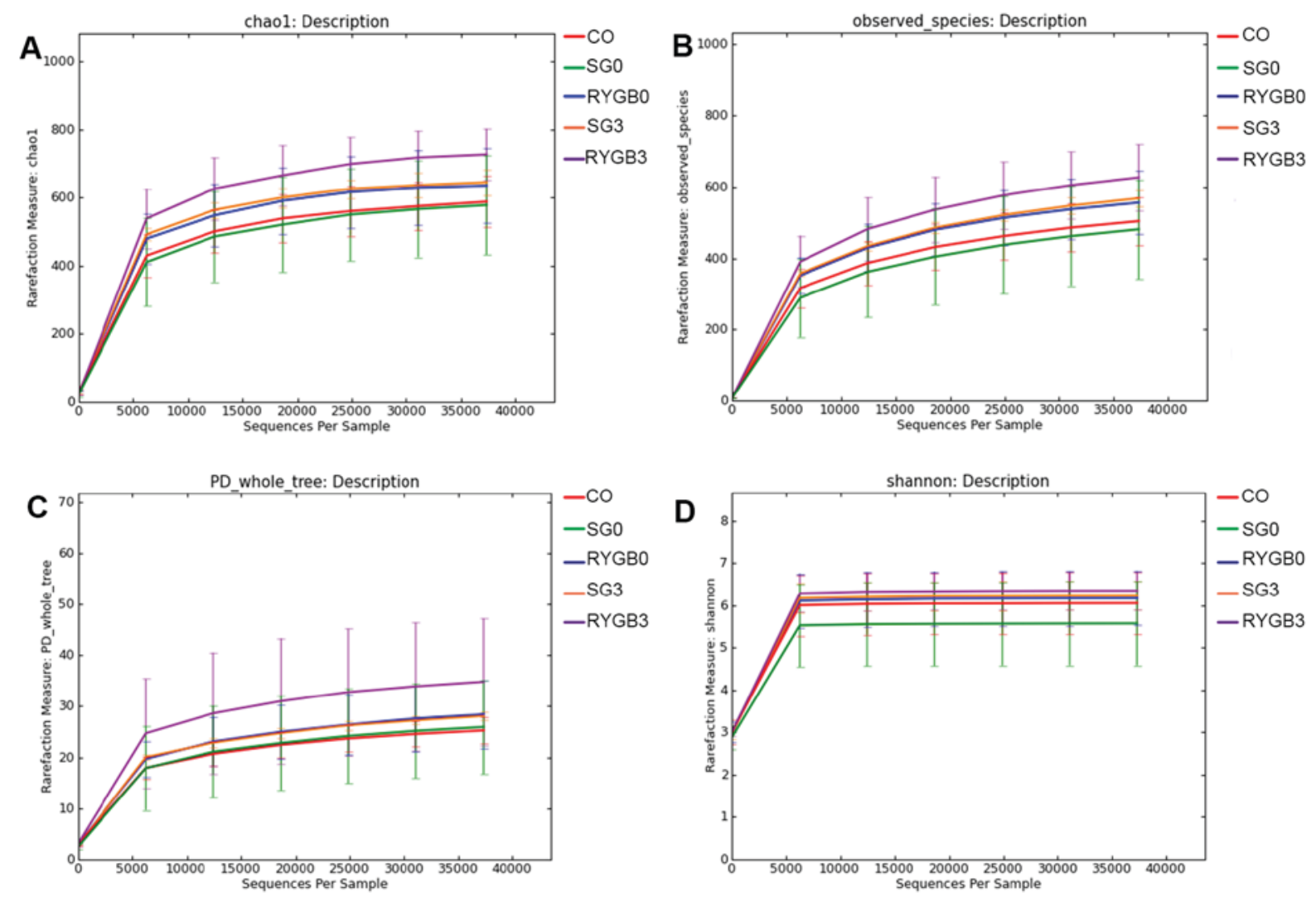

Figure 3. Rarefaction curves according to 4 different algorithms. (A) Chao 1. (B) Observed_species. (C) PD_whole_tree. (D) Shannon. The 5 lines of 5 groups gradually became flat, which indicates that the amount of sequencing data was appropriate. In other words, increasing the amount of sequencing would not lead to the discovery of any further species. The rarefaction curve also reflected the increased $\alpha$ diversity in the SG3 and RYGB3 groups compared with that in the SG0 and RYGB0 groups. CO, control group; SG, sleeve gastrectomy; RYGB, Roux-en-Y gastric bypass; SG0/3, group prior to/3 months after SG; $\mathrm{PD}$, phylogenetic diversity.
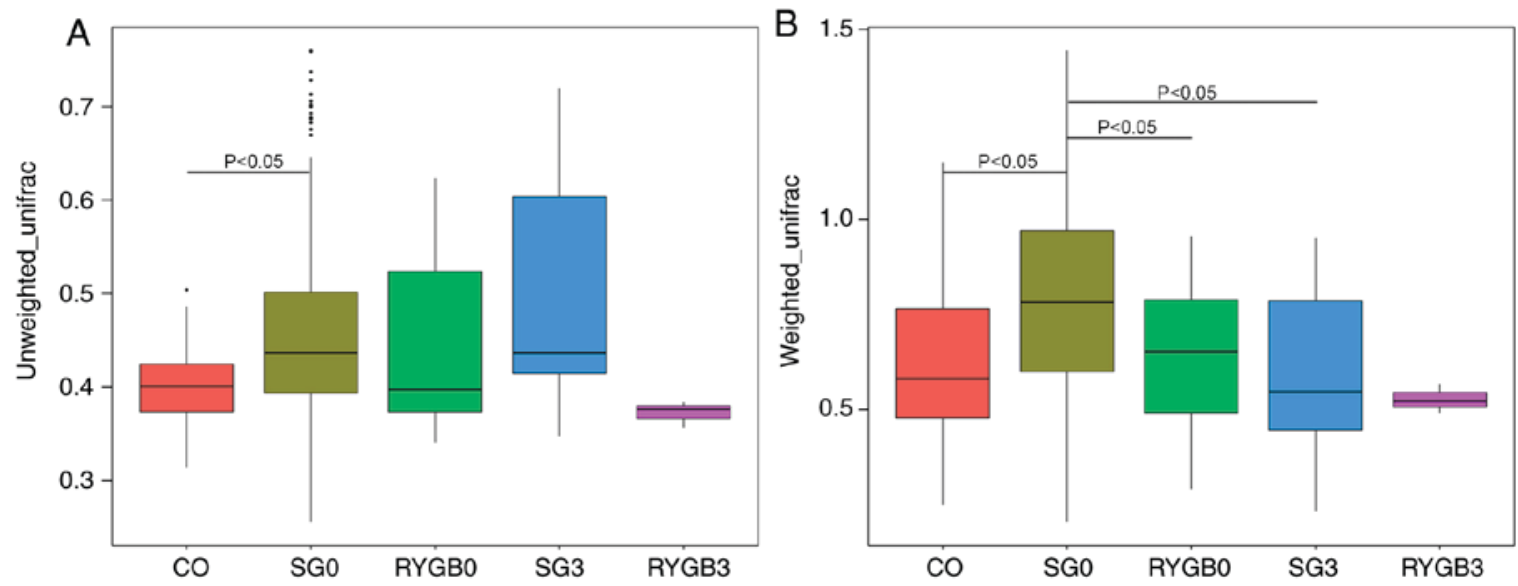

Figure 4. $\beta$ diversity of 5 groups using the ANOSIM algorithm. (A) Unweighted_unifrac and (B) weighted_unifrac. Analysis with unweighted_unifrac indicated an obviously higher $\beta$ diversity in the SG0 group compared with that in the CO group $(\mathrm{P}<0.05)$. According to the weighted_unifrac a clearly higher $\beta$ diversity was present in the SG0 group and the RYGB0 group compared with that in the CO group $(\mathrm{P}<0.05)$, as well as an obviously lower $\beta$ diversity in the SG3 group compared with that in the SG0 group $(\mathrm{P}<0.05)$. The horizontal lines inside the boxes indicate the median, whereas the lower lines and upper lines of the boxes indicate the 25th and the 75th percentiles, respectively. The upper and lower ends of the vertical lines represent the maximum and minimum points of data, respectively. The dots outside the boxes indicate the outliers (an observation point whose distance from mean value is greater than double standard deviations). CO, control group; SG, sleeve gastrectomy; RYGB, Roux-en-Y gastric bypass; SG0/3, group prior to/3 months after SG; PD, phylogenetic diversity.

SG3 group and the RYGB3 group deviated from that of the CO group, SG0 group and RYGB0 group. The plot of the SG3 group exhibited a converged tendency compared with that of the SG0 group (Fig. 5).
Species with statistically significant differences between groups. To identify the species with significant differences between groups at the phylogenetic level, the Kruskal-Wallis test was applied using LEfSe software. The species with 


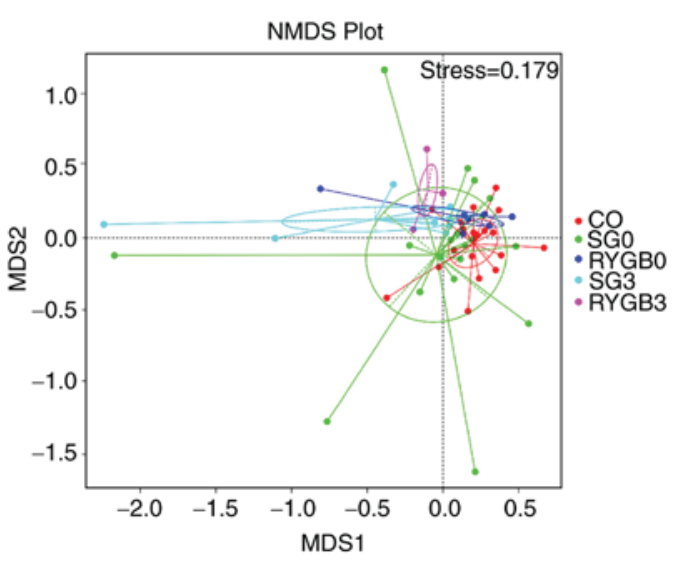

Figure 5. NMDS reduced-dimension graph. The community composition of the SG3 group and the RYGB3 group deviated from that of the CO group, SG0 group and the RYGB0 group. The plot of the SG3 group exhibited a converged tendency compared to the SG0 group. CO, control group; SG, sleeve gastrectomy; RYGB, Roux-en-Y gastric bypass; SG0/3, group prior to/3 months after SG; PD, phylogenetic diversity; NMDS, non-metric multidimensional scaling.

statistically significant differences are provided in Table III. The quantitative data with error bars are available in Fig. S1A-D.

Spearman correlation test. The Spearman correlation between the abundance of certain microbe and the BMI, as well as $\mathrm{GHb}$ and Glu were determined in all experimental groups (SG0, SG3, RYGB0 and RYGB3). The Spearman correlation analysis revealed that there were certain microbes whose abundance were positively or negatively correlated with BMI, $\mathrm{GHb}$ and Glu, indicating a counterproductive or beneficial effect, respectively, on surgical outcomes. The microbes with statistically significant differences are provided in Table IV. The correlation coefficients for each species are included in Table IV, Fig. S2 (phylum), Fig. S3 (class), Fig. S4 (order), Fig. S5 (family), Fig. S6 (genus) and Fig. S7 (species).

\section{Discussion}

The most effective therapy for treating morbid obesity and T2DM is bariatric surgery, and SG and RYGB are the most popular types of bariatric surgery procedures $(25,26)$. In the present study, patients receiving SG or RYGB achieved BMI reduction and T2DM remission; however, the mechanisms responsible for these outcomes in bariatric surgery remain to be fully elucidated. A recently published study suggested that the modulation of gut microbiota may be a possible factor (27).

$\alpha$ diversity is an index reflecting the variety of microbial species in stool samples. A higher $\alpha$ diversity indicates more species in one sample (7). In the present study, most of the $\alpha$ diversity results were consistent between the different algorithms applied, even though a small variation existed. Due to the different formulas and characteristics of the different algorithms, a small discrepancy was unavoidable (28). The present results indicated a similar $\alpha$ diversity between healthy volunteers and obese patients, which was consistent with the results of several cross-sectional studies $(29,30)$. Most previously published studies have observed a higher $\alpha$ diversity
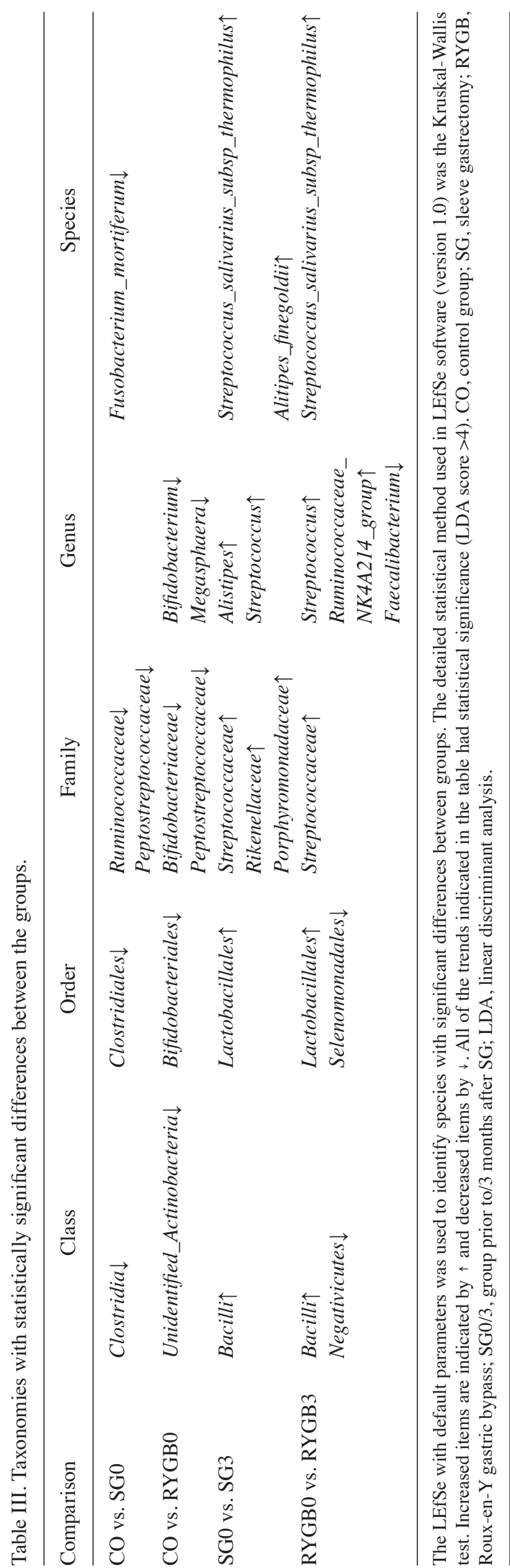


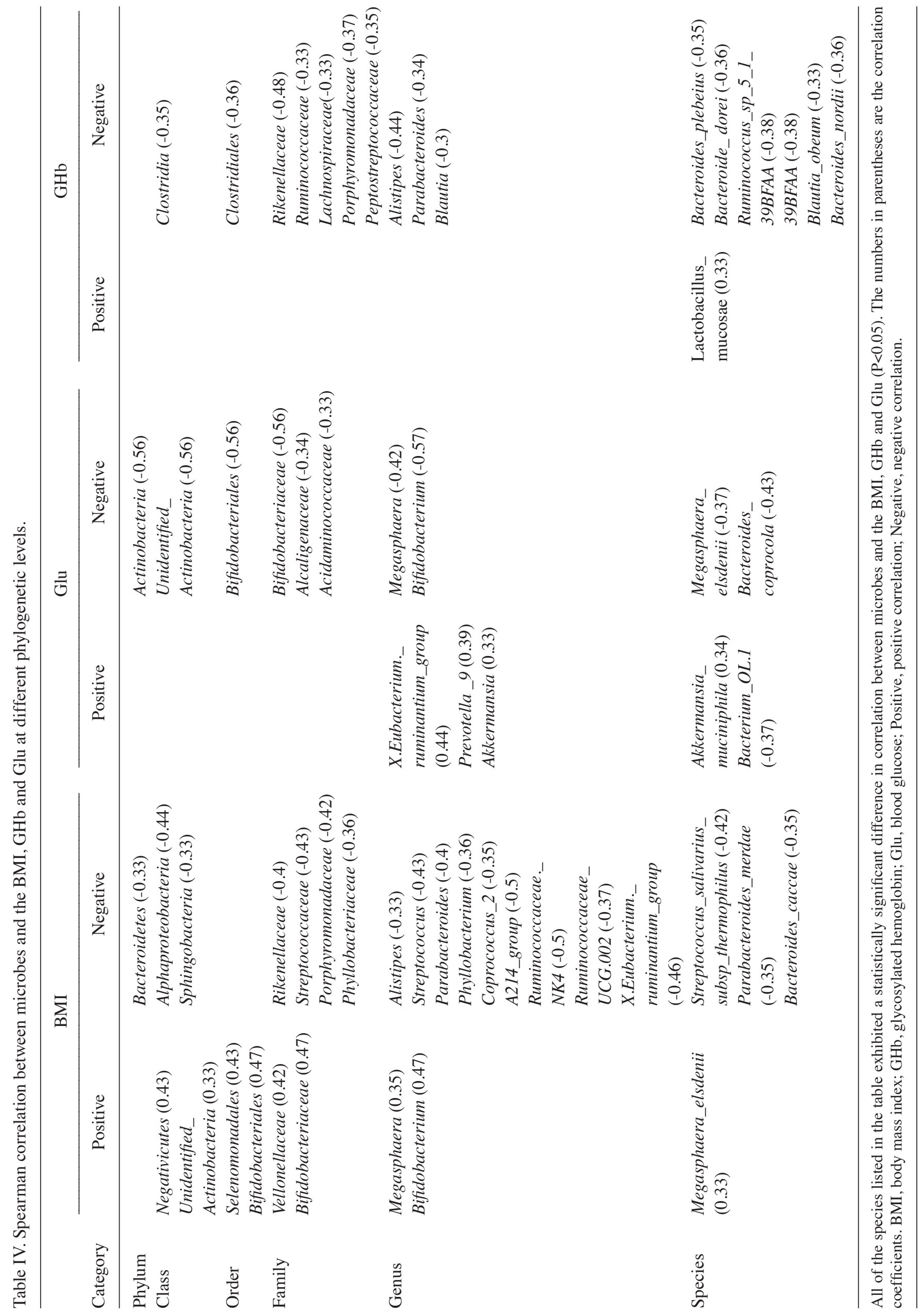


in healthy volunteers, but these studies did not provide any underlying causes of the alteration $(7,12,31)$. The current study did not stratify the T2DM patients into a separate group and as a result, it was impossible to assess the differences between T2DM patients and non-T2DM patients. The differences in gut microbiota between T2DM patients and non-T2DM patients should therefore be investigated in a future stuidies using a larger cohort. The present results indicated an increased $\alpha$ diversity after SG and RYGB, even though no statistical significance was obtained in the RYGB group ( $\mathrm{P}>0.05)$. The increase in $\alpha$ diversity after bariatric surgery was also observed by several similar studies $(7,15,28,32,33)$. While the mechanisms underlying the increase in $\alpha$ diversity after bariatric surgery remains elusive, it may be speculated that decreased gastric acid secretion and increased dissolved oxygen in the colon are a potential mechanism.

$\beta$ diversity is an index reflecting the heterogeneity of gut microbiota between samples in each group. A higher $\beta$ diversity is indicative of larger compositional differences in the gut microbiota between samples in a certain group (7). In the present study, a higher $\beta$ diversity was observed in obese patients compared with that in healthy volunteers, and after SG, the $\beta$ diversity decreased towards the level in the heathy volunteers. The results indicated that the heterogeneity of gut microbiota in obese patients is higher than that in heathy volunteers and decreased after bariatric surgery. In line with this, Liu et al (7) also determined a higher heterogeneity of the gut microbiota in obese patients compared with that in heathy volunteers by using the metagenome method. Only few studies have reported alterations in $\beta$ diversity after bariatric surgery. Medina et al (34) reported a decreased $\beta$ diversity after SG, but Guo et al (28) reported an unchanged $\beta$ diversity after SG in animal experiments. The community composition after bariatric surgery tended to deviate from that of patients without bariatric surgery, which indicated that bariatric surgery had a considerable influence on gut microbiota.

Previous studies on species with significant differences between healthy volunteers and obese patients with or without T2DM had a high heterogeneity. In the present study, significant differences in certain species at the phylogenetic level between healthy volunteers and obese patients with or without T2DM were identified. Most of these species belonged to the class of Clostridia. Of note, a reduction in Clostridia in the gut microbiota has been proven to be associated with obesity $(35,36)$. Certain species of Clostridia produce butyrate, the primary energy source of enterocytes (37). Furthermore, a reduction in butyrate production increases the permeability of enterocytes, leading to an increase in low-grade inflammation (38). The species producing butyrate may also cause satiety, thereby reducing dietary intake (39). Fusobacterium mortiferum is a species belonging to the Fusobacteria. In the present study, the decreased abundance of Fusobacterium mortiferum in obese patients was reported for the first time, to the best of our knowledge. Certain previous studies have reported an increased abundance of Fusobacteria after bariatric surgery $(15,28,40)$. In light of this, Fusobacteria may potentially be beneficial to obese patients. A decreased amount of actinobacteria was observed in obese patients compared with normal subjects in the present study. Bifidobacteriales is one of the representative orders among the actinobacteria. Several studies have reported a decrease in Bifidobacteriales in obese patients and T2DM patients $(41,42)$. Bifidobacteriales is also a butyrate producer (43) with the function of anti-endotoxaemia (44), which has been deemed one of the mechanisms of obesity.

Previous studies focusing on alteration of gut microbiota after bariatric surgery were highly heterogeneous. The present study observed a decrease in Negativicutes in the gut microbiota at 3 months after RYGB. Previously published studies have reported an increase in Negativicutes caused by a western high-fat diet in mice (45). To the best of our konwledge, the present study was the first to report a decrease in Selenomonadales after RYGB and an increase in Rikenellaceae after SG. A previous study has observed a reduced proportion of Rikenellaceae in patients with non-alcoholic fatty liver (46), which was, to a certain degree, consistent with the present results. An increase in the Ruminococcaceae NK4A214 group after bariatric surgery was also reported in the present study, results consistent with this (15) and opposite to this (11) have been reported, and the discrepancy between the different studies may be associated with differences in the surgical procedures applied. Previous studies reported a decrease in Faecalibacterium after RYGB $(15,40)$, which is consistent with the present results, but Furet et al (47) obtained an opposite result, i.e. a negative correlation between Faecalibacterium and low-grade inflammation. Streptococcus is part of the normal flora of the oral cavity, which may explain for the increases in Streptococcaceae, Streptococcus and Streptococcus salivarius subspecies thermophilus after RYGB or SG (48). Increases in Bacilli and Lactobacillales after bariatric surgery were observed in the present study; however, the detailed mechanism remains elusive.

The Spearman correlation between species and BMI, $\mathrm{GHb}$ and Glu in the experimental groups (SG0, RYGB0, SG3 and RYGB3) was assessed. The results suggested that the abundance of certain microbes at the phylogenetic level were positively correlated with the BMI, GHb or Glu, and that they appeared to have counterproductive effects on the outcomes of bariatric surgery. By contrast, the species whose abundance was negatively correlated with the BMI, GHb or Glu appeared to have beneficial effects on the outcomes of bariatric surgery. Of note, although no significant alterations of Akkermansiamuciniphilawere identified after bariatric surgery, a positive correlation between Akkermansia muciniphila and Glu was determined. Previous studies reported the beneficial effects of Akkermansia muciniphila on T2DM and obesity (49,50). The increase in Akkermansia muciniphila after bariatric surgery was deemed an important mechanism of the excellent outcomes of bariatric surgery $(40,51,52)$. The role of Akkermansia muciniphila requires to be explored as part of a rigorously designed study with a large sample size. The correlation coefficients ranged from 0.3 to 0.6 , which indicated a weak or moderate correlation between the abundance of species and the BMI, as well as GHb and Glu. No species with a high correlation were identified in the Spearman correlation analysis of the present study, and therefore, the present results should be interpreted with caution. Further studies are required to identify the correlation between the abundance of species and the BMI, GHb and Glu.

The following limitations of the study should be considered: i) The small sample size may have impaired the reliability of 
the results. ii) The healthy volunteers refused invasive testing; hence, it is not known whether any of them may have had undetected T2DM. However, this limitation influenced the results only to a small degree: The most important differential feature between the $\mathrm{CO}$ and the experimental group is not T2DM, but the BMI, and the volunteers in the CO group were all of normal weight. iii) The patients were not strictly paired between SG0 and SG3 or between RYGB0 and RYGB3 as certain patients were lost to follow up following bariatric surgery, which amplified the influence of individual microbial differences on the results. iv) The follow-up time was short (3 months after bariatric surgery) and the gut microbiota may have been unstable at that time. v) The inter-group differences in age and sex were important confounding factors that have affected the results of the current study and will need to be assessed in further research.

Bariatric surgery may cause obvious alterations in gut microbiota. Compared with healthy volunteers and obese patients without receiving bariatric surgery, the microbiota composition of post-bariatric surgery patients had unique characteristics. However, studies with a larger cohort and a longer follow-up may be required to confirm these results.

\section{Acknowledgements}

Not applicable.

\section{Funding}

This study was supported by the Beijing Municipal Commission of Science and Technology (grant nos. Z161100000116068).

\section{Availability of data and materials}

The data sets generated and analyzed during the study are available from the corresponding author upon reasonable request.

\section{Authors' contributions}

FGW collected the stools and the patient data. FGW, RXB, MY and WMY performed the next-generation 16s ribosomal DNA amplicon sequencing and statistical analysis. FGW was a major contributor in writing the manuscript. MMS and LYD conceived the study and revised the manuscript. All authors read and approved the final manuscript.

\section{Ethics approval and consent to participate}

All patients and healthy volunteers provided written informed consent, and the present study was approved by the ethics committee of Bejing Tiantan Hospital (Beijing, China).

\section{Patient consent for publication}

Not applicable.

\section{Competing interests}

The authors declare that they have no competing interests.

\section{References}

1. Schauer PR, Bhatt DL, Kirwan JP, Wolski K, Brethauer SA, Navaneethan SD, Aminian A, Pothier CE, Kim ES, Nissen SE, et al: Bariatric surgery versus intensive medical therapy for diabetes-3-year outcomes. N Engl J Med 370: 2002-2013, 2014

2. Colquitt JL, Pickett K, Loveman E and Frampton GK: Surgery for weight loss in adults. Cochrane Database Syst Rev: Cd003641, 2014.

3. Hayoz C, Hermann T, Raptis DA, Brönnimann A, Peterli R and Zuber M: Comparison of metabolic outcomes in patients undergoing laparoscopic roux-en-Y gastric bypass versus sleeve gastrectomy-a systematic review and meta-analysis of randomised controlled trials. Swiss Med Wkly 148: w14633, 2018.

4. Liaskos C, Koliaki C, Alexiadou K, Argyrakopoulou G, Tentolouris N, Diamantis T, Alexandrou A, Katsilambros N and Kokkinos A: Roux-en-Y gastric bypass is more effective than sleeve gastrectomy in improving postprandial glycaemia and lipaemia in non-diabetic morbidly obese patients: A short-term follow-up analysis. Obes Surg 28: 3997-4005, 2018.

5. Behary P and Miras AD: Food preferences and underlying mechanisms after bariatric surgery. Proc Nutr Soc 74: 419-425, 2015.

6. Thaler JP and Cummings DE: Minireview: Hormonal and metabolic mechanisms of diabetes remission after gastrointestinal surgery. Endocrinology 150: 2518-2525, 2009.

7. Liu R, Hong J, Xu X, Feng Q, Zhang D, Gu Y, Shi J, Zhao S, Liu W, Wang X, et al: Gut microbiome and serum metabolome alterations in obesity and after weight-loss intervention. Nat Med 23: 859-868, 2017.

8. Carlson AL, Xia K, Azcarate-Peril MA, Goldman BD, Ahn M, Styner MA, Thompson AL, Geng X, Gilmore JH and Knickmeyer RC: Infant gut microbiome associated with cognitive development. Biol Psychiatry 83: 148-159, 2018.

9. Kitai $\mathrm{T}$ and Tang WHW: Gut microbiota in cardiovascular disease and heart failure. Clin Sci (Lond) 132: 85-91, 2018.

10. Tang R, Jiang Y, Tan A, Ye J, Xian X, Xie Y, Wang Q, Yao Z and Mo Z: 16S rRNA gene sequencing reveals altered composition of gut microbiota in individuals with kidney stones. Urolithiasis 46: 503-514, 2018.

11. Kasai C, Sugimoto K, Moritani I, Tanaka J, Oya Y, Inoue H, Tameda M, Shiraki K, Ito M, Takei Y and Takase K: Comparison of the gut microbiota composition between obese and non-obese individuals in a Japanese population, as analyzed by terminal restriction fragment length polymorphism and next-generation sequencing. BMC Gastroenterol 15: 100, 2015.

12. Turnbaugh PJ,Hamady M, Yatsunenko T, Cantarel BL, Duncan A, Ley RE, Sogin ML, Jones WJ, Roe BA, Affourtit JP, et al: A core gut microbiome in obese and lean twins. Nature 457: 480-484, 2009.

13. Zhang H, DiBaise JK, Zuccolo A, Kudrna D, Braidotti M, Yu Y, Parameswaran P, Crowell MD, Wing R, Rittmann BE and Krajmalnik-Brown R: Human gut microbiota in obesity and after gastric bypass. Proc Natl Acad Sci USA 106: 2365-2370, 2009.

14. Tremaroli V, Karlsson F, Werling $\mathrm{M}$, Ståhlman M, Kovatcheva-Datchary P, Olbers T, Fändriks L, le Roux CW, Nielsen J and Bäckhed F: Roux-en-Y gastric bypass and vertical banded gastroplasty induce long-term changes on the human gut microbiome contributing to fat mass regulation. Cell Metab 22: 228-238, 2015.

15. Palleja A, Kashani A, Allin KH, Nielsen T, Zhang C, Li Y, Brach T, Liang S, Feng Q, Jørgensen NB, et al: Roux-en-Y gastric bypass surgery of morbidly obese patients induces swift and persistent changes of the individual gut microbiota. Genome Med 8: 67, 2016.

16. Sanmiguel CP, Jacobs J, Gupta A, Ju T, Stains J, Coveleskie K, Lagishetty V, Balioukova A, Chen Y, Dutson E, et al: Surgically induced changes in gut microbiome and hedonic eating as related to weight loss: Preliminary findings in obese women undergoing bariatric surgery. Psychosom Med 79: 880-887, 2017.

17. Griffith GW, Ozkose E, Theodorou MK and Davies DR: Diversity of anaerobic fungal populations in cattle revealed by selective enrichment culture using different carbon sources. Fungal Ecol 2: 87-97, 2009.

18. Martin M: Cutadapt removes adapter sequences from high-throughput sequencing reads. Embnet J 17,2011. doi: https://doi. org/10.14806/ej.17.1.200. 
19. Quast C, Pruesse E, Yilmaz P, Gerken J, Schweer T, Yarza P, Peplies J and Glöckner FO: The SILVA ribosomal RNA gene database project: Improved data processing and web-based tools. Nucleic Acids Res 41 (Database Issue): D590-D596, 2013.

20. Edgar RC, Haas BJ, Clemente JC, Quince C and Knight R: UCHIME improves sensitivity and speed of chimera detection. Bioinformatics 27: 2194-2200, 2011.

21. Haas BJ, Gevers D, Earl AM, Feldgarden M, Ward DV, Giannoukos G, Ciulla D, Tabbaa D, Highlander SK Sodergren E, et al: Chimeric 16S rRNA sequence formation and detection in Sanger and 454-pyrosequenced PCR amplicons. Genome Res 21: 494-504, 2011.

22. Edgar RC: UPARSE: Highly accurate OTU sequences from microbial amplicon reads. Nat Methods 10: 996-998, 2013.

23. Edgar RC: MUSCLE: Multiple sequence alignment with high accuracy and high throughput. Nucleic Acids Res 32: 1792-1797, 2004.

24. Segata N, Izard J, Waldron L, Gevers D, Miropolsky L, Garrett WS and Huttenhower C: Metagenomic biomarker discovery and explanation. Genome Biol 12: R60, 2011.

25. Buchwald H, Avidor Y, Braunwald E, Jensen MD, Pories W, Fahrbach K and Schoelles K: Bariatric surgery: A systematic review and meta-analysis. JAMA 292: 1724-1737, 2004.

26. Maggard MA, Shugarman LR, Suttorp M, Maglione M, Sugerman HJ, Livingston EH, Nguyen NT, Li Z, Mojica WA, Hilton L, et al: Meta-analysis: Surgical treatment of obesity. Ann Intern Med 142: 547-559, 2005.

27. Peck BCE and Seeley RJ: How does 'metabolic surgery' work its magic? New evidence for gut microbiota. Curr Opin Endocrino Diabetes Obes 25: 81-86, 2018.

28. Guo Y, Liu CQ, Shan CX, Chen Y, Li HH, Huang ZP and Zou DJ: Gut microbiota after Roux-en-Y gastric bypass and sleeve gastrectomy in a diabetic rat model: Increased diversity and associations of discriminant genera with metabolic changes. Diabetes Metab Res Rev 33, 2017. doi: 10.1002/dmrr.2857.

29. López-Contreras BE, Morán-Ramos S, Villarruel-Vázquez R, Macías-Kauffer L, Villamil-Ramírez H, León-Mimila P, Vega-Badillo J, Sánchez-Muñoz F, Llanos-Moreno LE, Canizalez-Román A, et al: Composition of gut microbiota in obese and normal-weight Mexican school-age children and its association with metabolic traits. Pediatr Obes 13: 381-388, 2018

30. Hakkak R, Korourian S, Foley SL and Erickson BD: Assessment of gut microbiota populations in lean and obese Zucker rats PLoS One 12: e0181451, 2017.

31. Yun Y, Kim HN, Kim SE, Heo SG, Chang Y, Ryu S, Shin H and Kim HL: Comparative analysis of gut microbiota associated with body mass index in a large Korean cohort. BMC Microbiol 17: 151,2017

32. Jahansouz C, Staley C, Bernlohr DA, Sadowsky MJ, Khoruts A and Ikramuddin S: Sleeve gastrectomy drives persistent shifts in the gut microbiome. Surg Obes Relat Dis 13: 916-924, 2017.

33. Kong LC, Tap J, Aron-Wisnewsky J, Pelloux V, Basdevant A, Bouillot JL, Zucker JD, Doré J and Clément K: Gut microbiota after gastric bypass in human obesity: increased richness and associations of bacterial genera with adipose tissue genes. Am J Clin Nutr 98: 16-24, 2013.

34. Medina DA, Pedreros JP, Turiel D, Quezada N, Pimentel F, Escalona A and Garrido D: Distinct patterns in the gut microbiota after surgical or medical therapy in obese patients. PeerJ 5: e3443, 2017.

35. Menni C, Jackson MA, Pallister T, Steves CJ, Spector TD and Valdes AM: Gut microbiome diversity and high-fibre intake are related to lower long-term weight gain. Int J Obes (Lond) 41: 1099-1105, 2017.

36. Nadal I, Santacruz A, Marcos A, Warnberg J, Garagorri JM, Moreno LA, Martin-Matillas M, Campoy C, Martí A, Moleres A, et al: Shifts in clostridia, bacteroides and immunoglobulin-coating fecal bacteria associated with weight loss in obese adolescents. Int J Obes (Lond) 33: 758-767, 2009.

37. Flint HJ, Duncan SH, Scott KP and Louis P: Interactions and competition within the microbial community of the human colon: Links between diet and health. Environ Microbiol 9: 1101-1111, 2007
38. Baothman OA, Zamzami MA, Taher I, Abubaker J and Abu-Farha M: The role of gut microbiota in the development of obesity and diabetes. Lipids Health Dis 15: 108, 2016

39. Xu J, Bjursell MK, Himrod J, Deng S, Carmichael LK, Chiang HC, Hooper LV and Gordon JI: A genomic view of the human-bacteroides thetaiotaomicron symbiosis. Science 299: 2074-2076, 2003

40. Graessler J, Qin Y, Zhong H, Zhang J, Licinio J, Wong ML $\mathrm{Xu} \mathrm{A}$, Chavakis T, Bornstein AB, Ehrhart-Bornstein M, et al: Metagenomic sequencing of the human gut microbiome before and after bariatric surgery in obese patients with type 2 diabetes: Correlation with inflammatory and metabolic parameters. Pharmacogenomics J 13: 514-522, 2013.

41. Santacruz A, Collado MC, García-Valdés L, Segura MT, Martín-Lagos JA, Anjos T, Martí-Romero M, Lopez RM, Florido J, Campoy C and Sanz Y: Gut microbiota composition is associated with body weight, weight gain and biochemical parameters in pregnant women. Br J Nutr 104: 83-92, 2010.

42. Schwiertz A, Taras D, Schäfer K, Beijer S, Bos NA, Donus C and Hardt PD: Microbiota and SCFA in lean and overweight healthy subjects. Obesity (Silver Spring) 18: 190-195, 2010.

43. Duncan SH, Belenguer A, Holtrop G, Johnstone AM, Flint HJ and Lobley GE: Reduced dietary intake of carbohydrates by obese subjects results in decreased concentrations of butyrate and butyrate-producing bacteria in feces. Appl Environ Microbiol 73: 1073-1078, 2007.

44. Cani PD, Neyrinck AM, Fava F, Knauf C, Burcelin RG, Tuohy KM, Gibson GR and Delzenne NM: Selective increases of bifidobacteria in gut microflora improve high-fat-diet-induced diabetes in mice through a mechanism associated with endotoxaemia. Diabetologia 50: 2374-2383, 2007.

45. Kim MS and Bae JW: Spatial disturbances in altered mucosal and luminal gut viromes of diet-induced obese mice. Environ Microbiol 18: 1498-1510, 2016

46. Del Chierico F, Nobili V, Vernocchi P, Russo A, Stefanis C, Gnani D, Furlanello C, Zandonà $\mathrm{A}$, Paci $\mathrm{P}$, Capuani $\mathrm{G}$, et al: Gut microbiota profiling of pediatric nonalcoholic fatty liver disease and obese patients unveiled by an integrated meta-omics-based approach. Hepatology 65: 451-464, 2017.

47. Furet JP, Kong LC, Tap J, Poitou C, Basdevant A, Bouillot JL, Mariat D, Corthier G, Doré J, Henegar C, et al: Differential adaptation of human gut microbiota to bariatric surgery-induced weight loss: Links with metabolic and low-grade inflammation markers. Diabetes 59: 3049-3057, 2010.

48. Wang K, Lu W, Tu Q, Ge Y,He J,Zhou Y, Gou Y, Van Nostrand JD, Qin Y, Li J, et al: Preliminary analysis of salivary microbiome and their potential roles in oral lichen planus. Sci Rep 6: 22943 , 2016.

49. Everard A, Belzer C, Geurts L, Ouwerkerk JP, Druart C, Bindels LB, Guiot Y,Derrien M, MuccioliGG,Delzenne NM, et al: Cross-talk between Akkermansia muciniphila and intestinal epithelium controls diet-induced obesity. Proc Natl Acad Sci USA 110: 9066-9071, 2013

50. Shin NR, Lee JC, Lee HY, Kim MS, Whon TW, Lee MS and Bae JW: An increase in the Akkermansia spp. population induced by metformin treatment improves glucose homeostasis in diet-induced obese mice. Gut 63: 727-735, 2014

51. Liou AP, Paziuk M, Luevano JM Jr, Machineni S, Turnbaugh PJ and Kaplan LM: Conserved shifts in the gut microbiota due to gastric bypass reduce host weight and adiposity. Sci Transl Med 5: 178ra14, 2013.

52. Yan M, Song MM, Bai RX, Cheng S and Yan WM: Effect of Roux-en-Y gastric bypass surgery on intestinal Akkermansia muciniphila. World J Gastrointest Surg 8: 301-307, 2016.

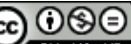

This work is licensed under a Creative Commons Attribution-NonCommercial-NoDerivatives 4.0 International (CC BY-NC-ND 4.0) License. 